\title{
Automatic Detection of Small Surface Targets with Electro-Optical Sensors in a Harbor Environment
}

\author{
Henri Bouma*, Dirk-Jan J. de Lange, Sebastiaan P. van den Broek, \\ Rob A.W. Kemp and Piet B.W. Schwering; \\ TNO Defence, Security and Safety, P.O. Box 96864, 2509 JG The Hague, The Netherlands
}

\begin{abstract}
In modern warfare scenarios naval ships must operate in coastal environments. These complex environments, in bays and narrow straits, with cluttered littoral backgrounds and many civilian ships may contain asymmetric threats of fast targets, such as rhibs, cabin boats and jet-skis. Optical sensors, in combination with image enhancement and automatic detection, assist an operator to reduce the response time, which is crucial for the protection of the naval and land-based supporting forces. In this paper, we present our work on automatic detection of small surface targets which includes multi-scale horizon detection and robust estimation of the background intensity. To evaluate the performance of our detection technology, data was recorded with both infrared and visual-light cameras in a coastal zone and in a harbor environment. During these trials multiple small targets were used. Results of this evaluation are shown in this paper.
\end{abstract}

Keywords: Detection, small targets.

\section{INTRODUCTION}

In modern warfare scenarios naval ships must operate in coastal environments; in bays and narrow straits. These complex environments with cluttered littoral backgrounds and many civilian ships may contain asymmetric threats of fast targets, such as rhibs, cabin boats and jet-skis. Optical sensors, in combination with image enhancement and automatic detection, assist an operator to reduce the response time, which is crucial for the protection of the naval and land-based supporting forces.

TNO is examining several aspects of the use of electro-optical systems in the research programme "Electro-optical sensor systems" for the Dutch Ministry of Defence. The system should be able to detect and classify surface targets in maritime environments. Our system concept consists of an image processing chain [17], which includes components that perform image enhancement [14], automatic detection, clutter reduction and classification [3].

In this paper, we present our work on automatic detection of surface targets which includes multi-scale horizon detection and robust estimation of the background intensity. To evaluate the performance of our detection technology, data was recorded with both infrared and visual-light cameras in a coastal zone and in a harbor environment. During these trials multiple small targets were used. Results of the evaluation are shown in this paper.

This paper is organized as follows: the detection method is described in Section 2, the experiments and results are presented in Section 3. Finally, the conclusions and discussion can be found in Section 4.

* henri.bouma@tno.nl; phone +31.70.374.0399; fax +31.70.374.0653

Electro-Optical Remote Sensing, Photonic Technologies, and Applications II,

edited by Gary W. Kamerman, Ove K. Steinvall, Keith L. Lewis, Thomas J. Merlet, Richard C. Hollins

Proc. of SPIE Vol. 7114, 711402 - (c) 2008 SPIE · CCC code: 0277-786X/08/\$18 - doi: 10.1117/12.799813

Proc. of SPIE Vol. $7114711402-1$ 


\section{METHOD}

Our method for the automatic detection of surface targets in a maritime environment consists of three steps: horizon detection, background estimation and object detection (Figure 1).

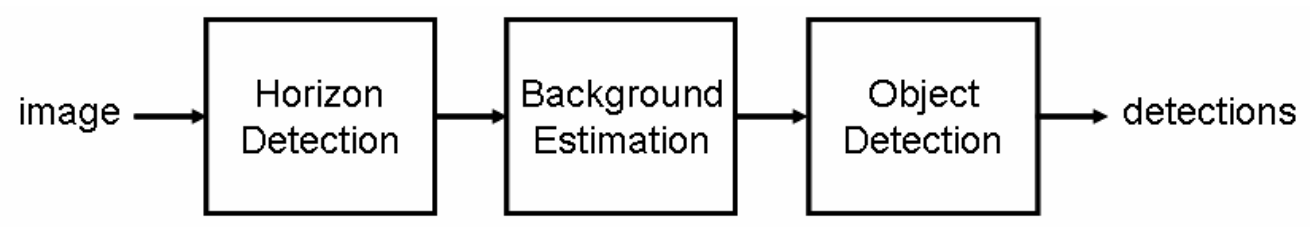

Figure 1: The method consists of three steps: horizon detection, background estimation and object detection.

\subsection{Horizon detection}

In the first step of our method the location and orientation of the horizon are detected. The horizon is used for stabilization, background estimation, separation between false detections above the horizon and surface targets below the horizon, and to compute features for classification [3].

Our system enables the use of gray-value images of a visual light, near infrared (NIR), mid-wave infrared (MW-IR) or long-wave infrared (LW-IR) cameras. We did not use color information or stereo information in the steps of our method.

In literature, basically two approaches can be distinguished: region-based and edge-based approaches.

Naive region-based approaches will fail in our images. These methods are often based on the model of two Gaussians that describe the distributions of the intensities of the sky and the ground region. Examples of this approach for horizon detection are based on covariance matrices and eigenvalues [8,9], linear discriminant analysis and the Mahalanobis distance [21] and two Gaussian models [6]. These methods will fail on our gray-value images from different cameras. The intensity of the sea and the air are often slowly varying. Therefore, the intensities in a region cannot be modeled by one constant value and additive noise. The region-based segmentations may be improved by using multiple classes, multiple scales or texture $[20,21]$.

The easiest way to separate different regions that each have a slowly varying intensity is to use the edges of these regions. Edge detectors are very suitable because they mainly respond to local changes. They are often based on methods of Sobel, Prewitt, Roberts and Canny [12]. Examples of edge-based methods for horizon detection are based on thinning, edge grouping, Kalman filtering and multiple-hypothesis tracking [4], least squares regression, splitting with chi-squared tests and merging with the Akaike information criterion [5] and the Hough transform [7]. Sheng et al. [18] used edge focusing for horizon detection and they used a curve saliency measure to favor long over short curves and smooth over wiggly curves. Edge focusing requires Gaussian derivatives at multiple scales to construct a scale space. Although there exist fast and accurate implementations [1], the computation of them on the whole image at multiple scales remains expensive in image sequences.

We used a very fast multi-scale [11] edge detection technique. We assume that the horizon will appear approximately as a straight line in the image and that there is a local intensity difference between the sea surface below the horizon and the air (or coast) above the horizon (or coast line). Examples of horizon detection are shown in the Figures 2 and 3. 


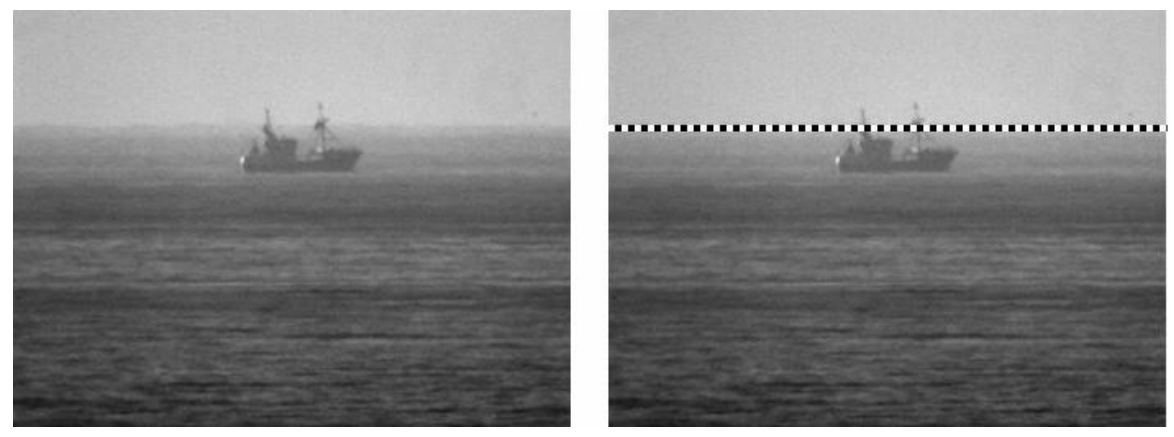

Figure 2: Horizon detection (dashed line in the right image) is possible despite other horizontal patterns.
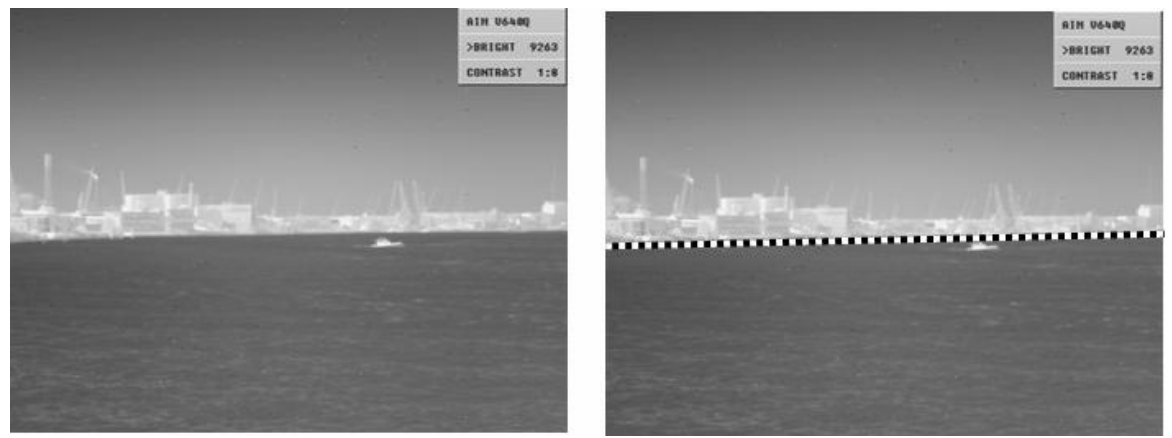

Figure 3: Our horizon detector is able to find the boundary between water and a coast or quay in a harbor.

\subsection{Background estimation}

In the second step of our method the background intensity of the image is estimated. The background intensity is used by object detection, which uses the intensity difference between background (the sea) and foreground (the object). Subtraction of the background intensity from input image removes variation in the background (Figure 4), and simplifies object detection.
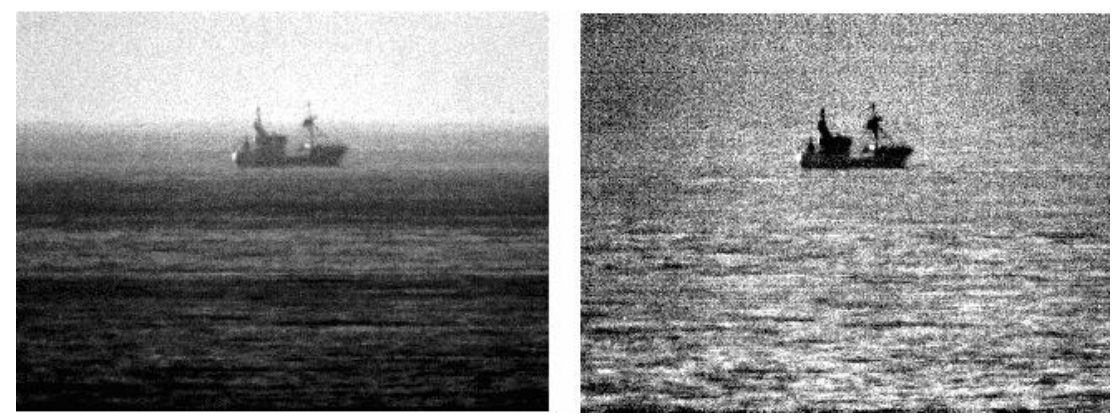

Figure 4: Horizontal patterns are removed by the background estimation.

Van den Broek et al. [2] described a system for the estimation of the background intensity in MW-IR and LW-IR images. The system computed the average and the standard deviation for each row. Subsequently, two continuous models defined by a few parameters - were fitted to the average and the standard deviation. This approach has two disadvantages. First, the continuous model is not flexible enough to describe realistic intensity variations in the background in NIR or visual-light images. An example where the model would have failed is shown in Figure 4, where the intensity of the sea surface cannot be modeled by a third-order polynomial and a logarithmic function. Second, the average is not a robust estimator of the background intensity (and therefore a fitted continuous model was needed to make it more robust). An average-based estimation of the background intensity is influenced by foreground objects and clutter. An example where an average would fail - and where even the median fails - is shown in Figure 5 (left). To obtain a more robust and flexible estimation of the background intensity, we used a robust estimator [15] without the fit of a polynomial model. 


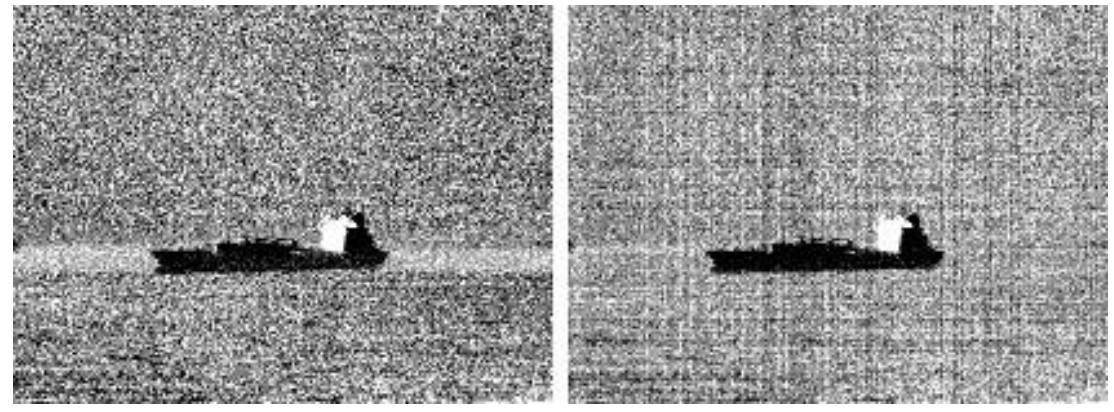

Figure 5: A background estimator based on the median will lead to artifacts (left). Our robust estimator is not hampered by objects in the image (right).

To estimate the intensity of the background, we could not easily have used a low-pass filter, because the background includes sharp transitions (e.g., near the horizon). Neither could we simply have used the basic operations from mathematical morphology, because of the variety of object size and the variation in background intensity. The only assumption about object size that we made is that the background region in an image is larger than the object region.

Robust estimation of the background intensity may be hampered by many aspects that do not occur in perfect synthetic images, but that will cause failure of the system on representative images. Therefore, we used data from several trials, including data from SURFER [2], SPITS [19], and the Rotterdam harbor [14]. This data contains information from different weather conditions, open sea and harbor environment, and several imaging artifacts.

Our background-estimation step uses information about the orientation of the image, which is supplied by the horizon detection step. If the orientation of the image is not taken into account, the background intensity is not estimated correctly near the horizon (Figure 6). A rotation can be performed to align the horizon with the rows in the image. However, a rotation may suffer from artifacts near the boundary or artifacts due to interlacing. Our method for the robust estimation of the background intensity does not suffer from these artifacts (Figure 7).
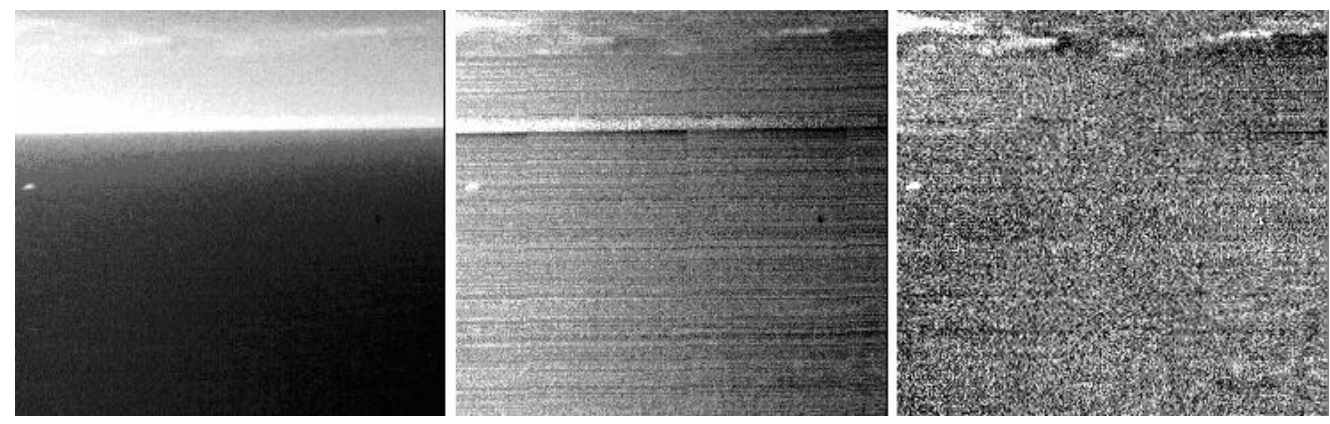

Figure 6: Orientation of the horizon. If the orientation of the input image (left) is not taken into account, the contrast near the horizon is not near zero (center). If it is taken into account, the contrast is almost zero (right).
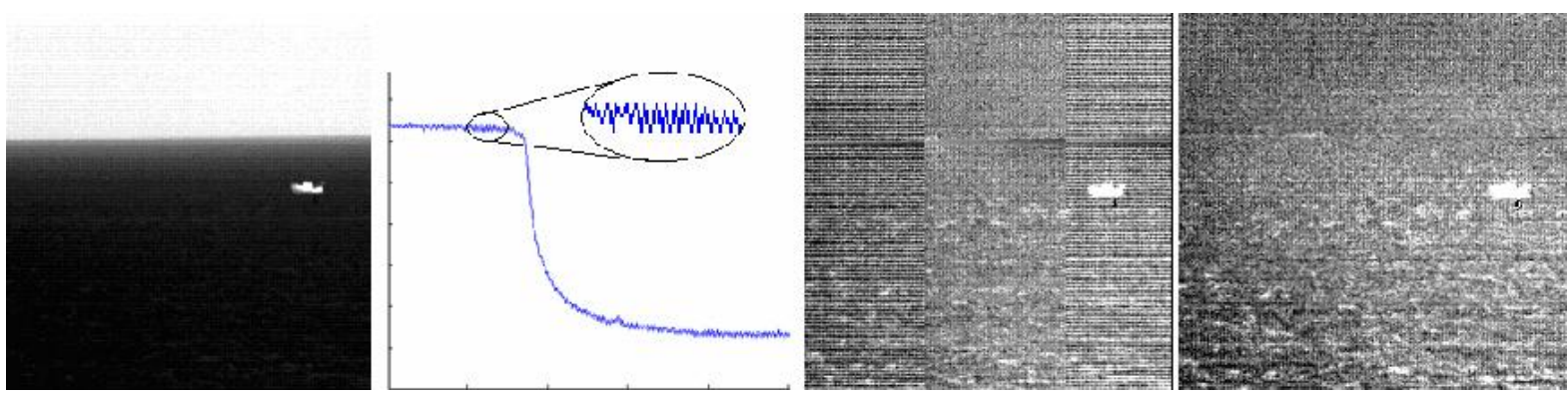

Figure 7: Orientation and interlacing. Input image (left) with an intensity difference between even and odd rows (center-left) can cause artifacts in the contrast image when it is rotated (center-right). The artifacts are removed by taking interlacing into account. 
On one hand, there is an intensity difference in the vertical direction between the air and the sea region (top and bottom of the image). However, there are also other patterns in other directions, e.g., caused by the sun, vignetting or narcissus. For a correct background estimation and to avoid unstable behavior, these variations in the other direction must be taken into account (Figure 8 and 9).
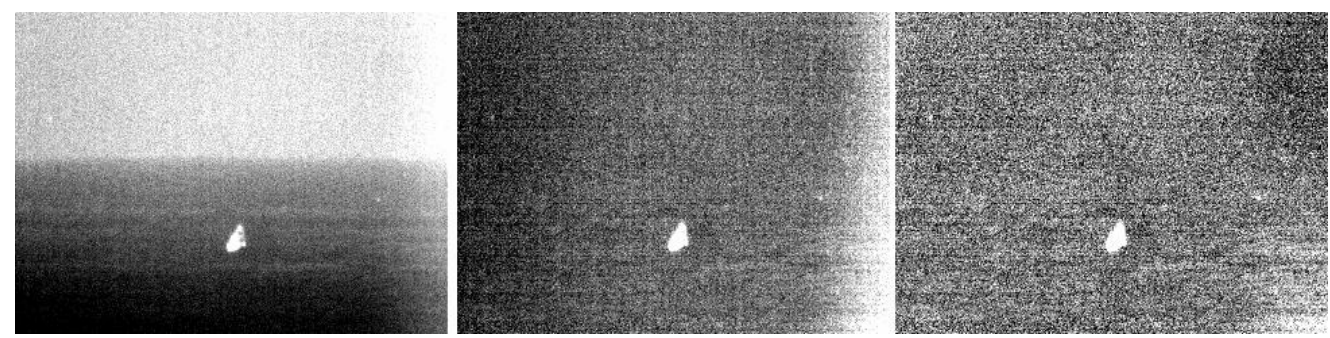

Figure 8: Input image with variation from left to right (left), contrast image where the variation caused by the camera has not been removed (center) and contrast image where the variation has been removed (right).
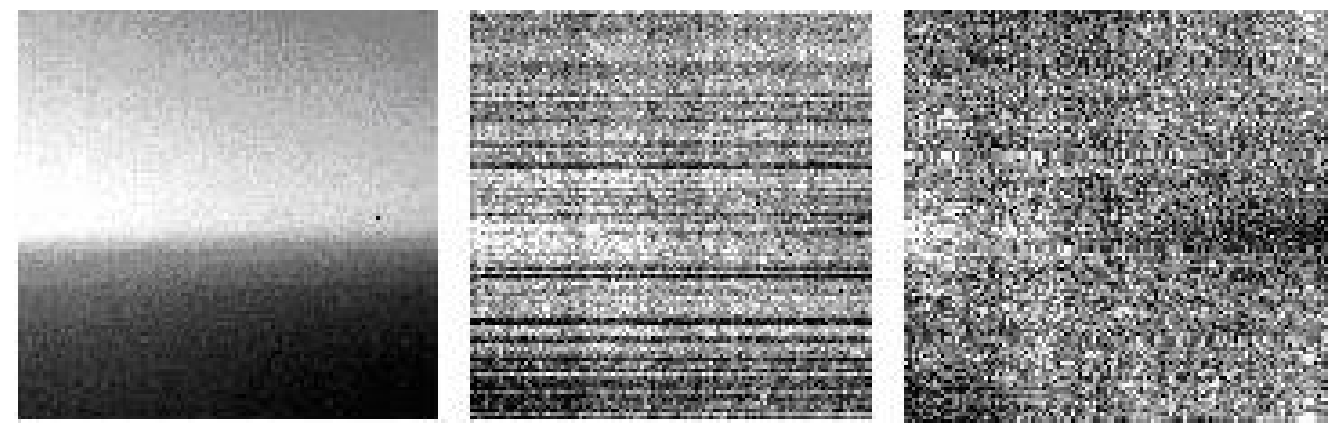

Figure 9: Removal of (most of) the variation from left to right helps the robust estimator to remove variation in the vertical direction without serious artifacts. Input (left), artifacts introduced by a robust estimator (center), corrected (right).

Robust estimation may lead to quantization artifacts in digital images and non-optimal removal of the background intensity. Our estimator has solved the problems with these artifacts (Figure 10).
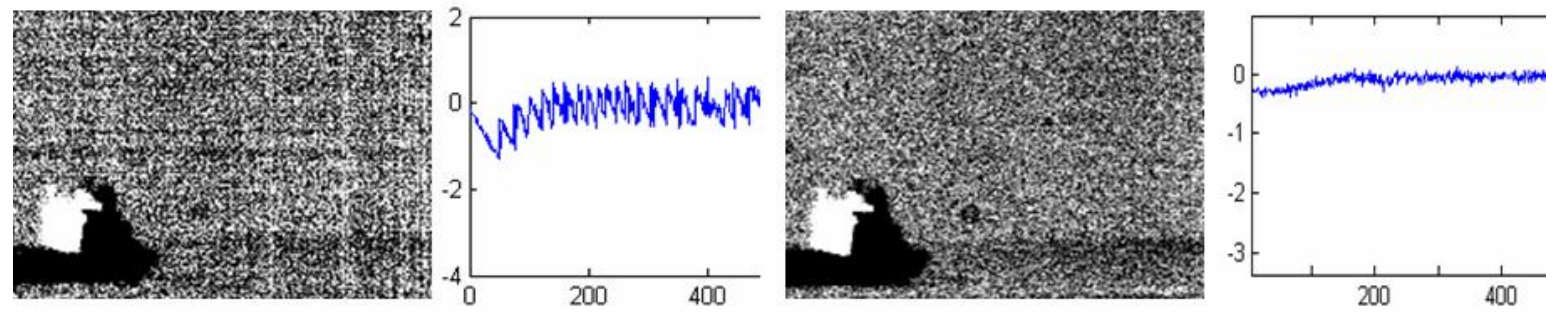

Figure 10: Quantization. Robust estimation may easily lead to quantization artifacts in digital images (left). Our background estimation method has solved the problem with these artifacts (right).

\subsection{Object detection}

In the third step of our method the objects are detected. Object detection uses the intensity difference between background and the foreground. After background estimation, subtraction and normalization the objects are detected with hysteresis thresholding (Figure 11 and Figure 12). Note that the automatic detection was performed with the same parameters, although we used images from different cameras and objects of different sizes. 

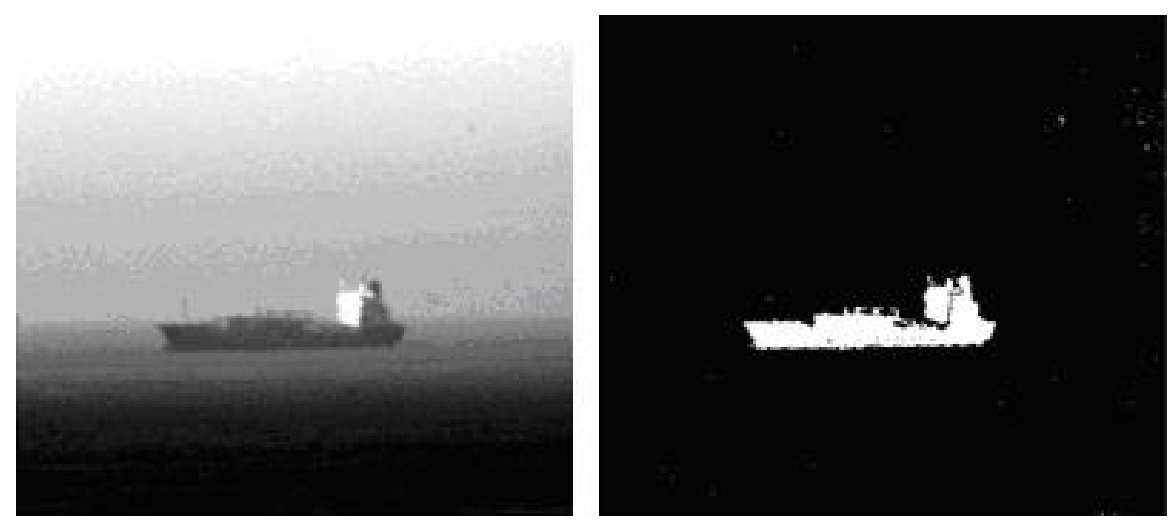

Figure 11: Detection of a large ship.
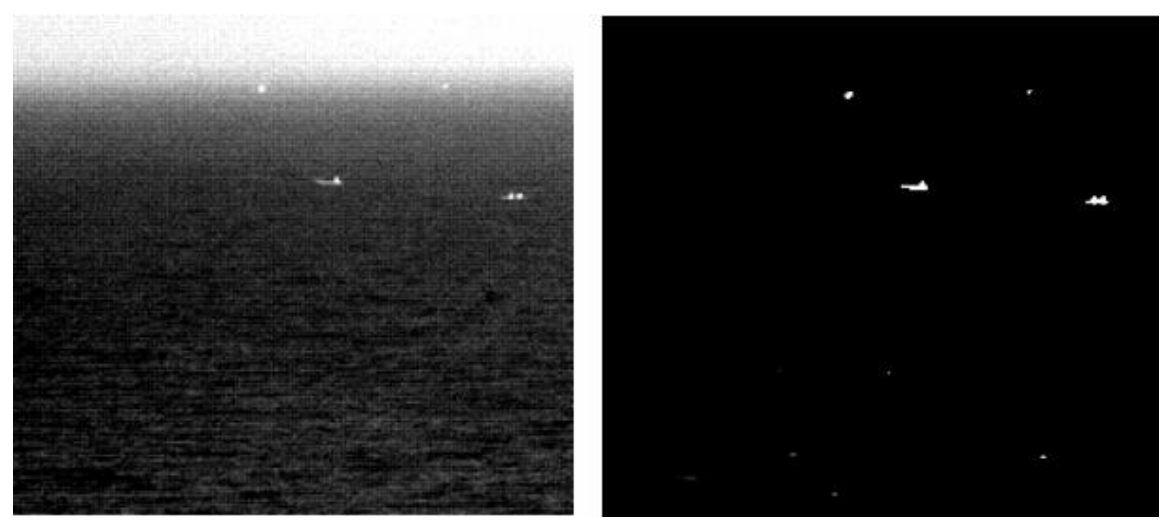

Figure 12: Detection of four small targets; two buoys, a zodiac and a 2-person kayak (zodiac and kayak at 1250 meter).

\section{EXPERIMENTS AND RESULTS}

In our experiments, we used four categories of data (Table 1) that were all processed with the same parameter settings.

Table 1: Description of the data used in our experiments.

\begin{tabular}{|c||c|c|c|c|c|}
\hline Data & Camera & No. sequences & Target size & Examples of clutter & Clutter \\
\hline \hline 1 & MW & 10 & small & waves, sun, clouds, coast & Low \\
\hline 2 & NIR & 7 & small and large & waves, sun, clouds, coast, patterns & Medium \\
\hline 3 & LW,MW,NIR,Visual & 6 & - & waves, sun, clouds, coast & High \\
\hline 4 & LW,MW,NIR,Visual & 6 & small and large & waves, sun, clouds, harbor & Medium \\
\hline
\end{tabular}

The results for horizon detection and object detection are presented in Table 2 . In more than $80 \%$ of the sequences, the horizon is detected correctly; i.e. with a distance between the automatically detected horizon and a manually annotated horizon less than 5 pixels. An example where horizon detection fails is shown in Figure 13. This figure shows a dark region at the bottom of the image with an edge that matches a straight line over the entire width of the image.

Table 2 also shows the detection sensitivity, the number of false alarms per frame and the percentage of frame area that was covered by false alarms (Figure 14 shows an example). For comparison, results of a previous implementation are included between parentheses. The previous implementation was mainly designed for LW-IR and MW-IR images only. The results show that the current version can also be applied to cameras with other wavelengths and that our system performs better than the previous implementation. The algorithm is more robust and more generally applicable. On the datasets 3 and 4 we only counted the detections below the automatically found horizon to eliminate false detections due 
to the cluttered coast and harbor. The sensitivity of the fourth dataset would have been higher if the horizon detection would not have failed in $17 \%$ of the cases.

Table 2: Results for horizon detection and object detection. For comparison, the results of a previous implementation [2] are shown between parentheses "()". For the datasets 3 and 4, only detections below the automatically detected horizon (coast line) were used to avoid clutter caused by the coast or the harbor.

\begin{tabular}{|c||c||c|c|c|c|}
\hline \multirow{2}{*}{ Data } & Correct horizon & Detection & \multicolumn{2}{|c|}{ No. of false alarms / frames } & Area false alarms / frame size \\
\cline { 4 - 6 } & & sensitivity & average & median & average \\
\hline \hline 1 & $90 \%$ & $\mathbf{9 3}(93) \%$ & $1.9(4.3)$ & $\mathbf{1 . 0}(3.5)$ & $0.2(20) \%$ \\
\hline 2 & $60 \%$ & $\mathbf{1 0 0}(88) \%$ & $0.4(297)$ & $\mathbf{0 . 0}(150)$ & $0.0(3.4) \%$ \\
\hline 3 & $100 \%$ & - & $2.2(11)$ & $\mathbf{1 . 0}(2.0)$ & $0.1(0.8) \%$ \\
\hline 4 & $83 \%$ & $\mathbf{8 5}(51) \%$ & $11(12)$ & $\mathbf{3 . 2}(7.1)$ & $0.2(2.5) \%$ \\
\hline
\end{tabular}

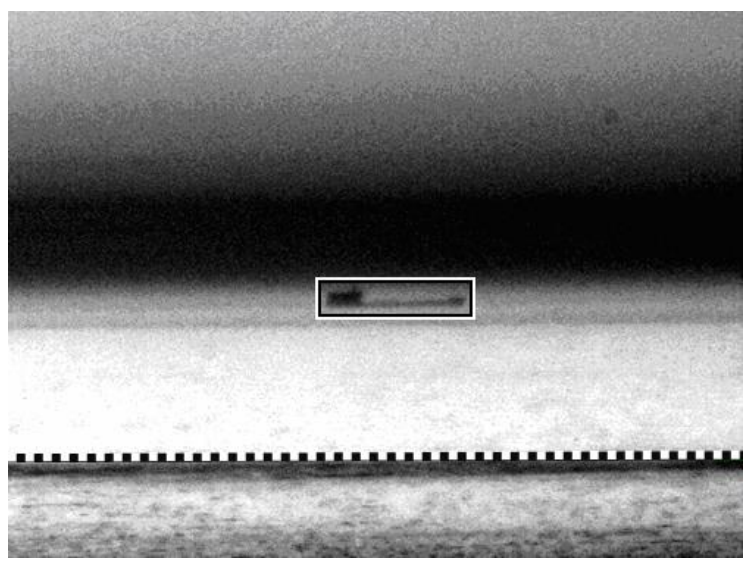

Figure 13: Example from dataset 2 where horizon detection fails due to a dark horizontal pattern. The automatically detected horizon is indicated with a dashed line.
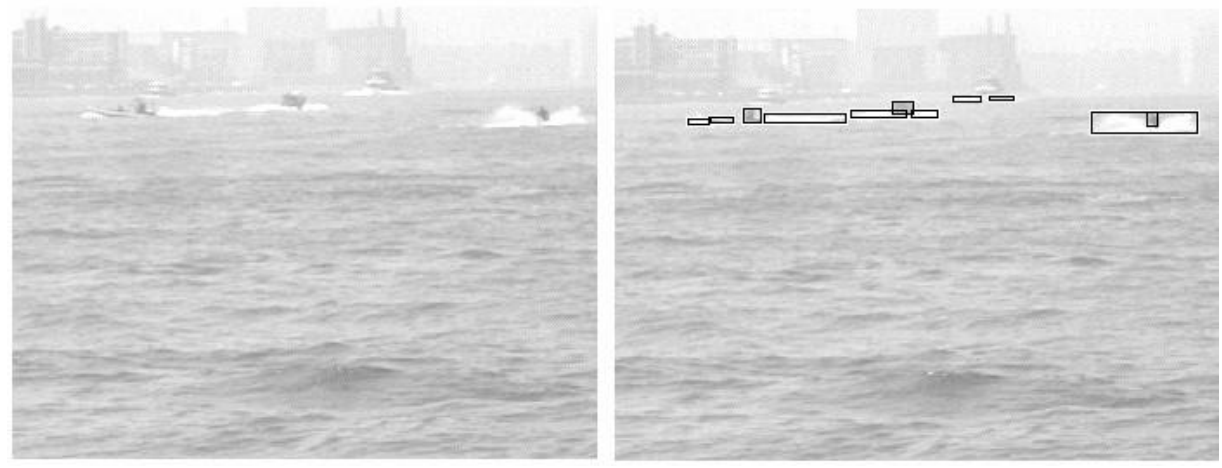

Figure 14: Example of object detection from dataset 4. The automatic detections are indicated with boxes.

\section{CONCLUSIONS AND DISCUSSION}

In this paper, we presented our work on automatic detection of small surface targets which includes multi-scale horizon detection and robust estimation of the background intensity. The system was evaluated on infrared and visual-light images in a coastal zone and in a harbor environment and multiple small and large targets were used. The results showed that horizon detection is accurate in more than $80 \%$ of the sequences. Object detection sensitivities were above $80 \%$ at $3 \%$ (or less) false detections per frame in cluttered data. 


\section{ACKNOWLEDGEMENTS}

The work for this paper was supported by the Netherlands Ministry of Defence under the programme V602 "Electrooptical sensor systems".

\section{REFERENCES}

1. H. Bouma, A. Vilanova, J. Olivan Bescos, B.M. ter Haar Romeny and F.A. Gerritsen, "Fast and Accurate Gaussian Derivatives based on B-Splines", Proc. SSVM, LNCS 4485, pp. 406-417, May 2007.

2. S.P. van den Broek, E.J. Bakker, D.J.J. de Lange and A. Theil, "Detection and classification of infrared decoys and small targets in a sea background", Proc. SPIE Targets and Backgrounds, vol. 4029, pp. 70-80, 2000.

3. S.P. van den Broek, H. Bouma, M.A.C. Degache, "Discriminating small extended targets at sea from clutter and other classes of boats in infrared and visual light imagery", Proc. SPIE Defense and Security, vol. 6969, 2008.

4. I. J. Cox, J. M. Rehg and S. L. Hingorani, "A Bayesian Multiple Hypothesis Approach to Contour Segmentation”, Int. J. of Computer Vision, 11, 1, 5-24, 1993.

5. F. Cozman and E. Krotkov, "A line linker based on Frequentist Statistics tools: Splitting lines with Chi-square tests and merging lines with the Akaike Information Criterion", Technical report, Carnegie Mellon University, 1996.

6. C. Demonceaux, P. Vasseur and C. Pegard, Omnidirectional vision on UAV for attitude computation, IEEE Int. Conf. Robotics and Automation, 2006.

7. D. Dusha, W. Boles and R. Walker, "Attitude Estimation for a Fixed-Wing Aircraft Using Horizon Detection and Optical Flow", Proc. Digital Image Computing Techniques and Applications, pages pp. 485-492, Australia, 2007.

8. S.M. Ettinger, Design and implementation of autonomous vision-guided micro air vehicles, M.Sc. Thesis, Univ. of Florida, USA, 2001.

9. S. M. Ettinger, M. C. Nechyba, P. G. Ifju and M. Waszak, "Towards Flight Autonomy: Vision-Based Horizon Detection for Micro Air Vehicles", Florida Conf. Recent Advances in Robotics, May 2002.

10. T.G. McGee, R. Sengupta and J.K. Hedrick "Obstacle Detection for Small Autonomous Aircraft Using Sky Segmentation", Proc. IEEE Int. Conf. Robotics and Automation, 2005.

11. B.M. ter Haar Romeny, "Front-end vision and multi-scale image analysis: computer vision theory and applications", Springer, 2003.

12. F. van der Heijden, "Image based measurement systems: Object Recognition and parameter estimation", J. Wiley and Sons, 1995.

13. J. Kellogg, C. Bovais et al., "The NRL MITE Air Vehicle", Proc. Int. Conf. Unmanned Air Vehicle Systems, 2001.

14. R.A.W. Kemp, J.F. de Groot, S.P. van den Broek, D.J.J. de Lange, J. Dijk, P.B.W. Schwering, "Results of optical detection trials in harbour environment", Proc. SPIE Defense and Security, vol. 6943, 2008.

15. P. Meer, C.V. Stewart, and D.E. Tyler, "Robust Computer Vision: An Interdisciplinary Challenge", J. Computer Vision and Image Understanding, vol. 78, nr. 1, pp. 1-7, 2000.

16. M. Ollis and A. Stentz, "Vision-Based Perception for an Automated Harvester", Proc. IEEE Int. Conf. Intelligent Robots and Systems, vol. 3, pp. 1838-1844, 1977.

17. P.B.W. Schwering, S.P. van den Broek and M. van Iersel, "EO System Concepts in the Littoral", Proc. SPIE Defence and Security, vol. 6542, 2007.

18. Y. Sheng, X. Yang, D. McReynolds, Z. Zhang, L. Gagnon and L. Sévigny, "Real-world multisensor image alignment using edge focusing and Hausdorff distances", Proc. SPIE Sensor fusion, vol. 3719, 1999.

19. A. Theil, A.G. Huizing, A.W.P. van Heijningen, "Single Picture Integration for Territorial Water Surveillance (SPITS): An initiative to improve situational awareness in littoral waters", Maritime systems and technology (MAST) conference, France, 2006.

20. S. Todorovic and M.C. Nechyba, "A vision system for horizon tracking and object recognition for micro air vehicles", Proc. Florida Conf. on Recent Advances in Robotics, 2004.

21. S. Todorovic and M.C. Nechyba, "A vision system for intellignet mission profiles of Micro Air Vehicles", IEEE Trans. Vehicular Technology, vol. 53, no. 6, pp. 1713-1725, 2004.

22. P.J. Withagen; K. Schutte; A.M. Vossepoel; M.G.J. Breuers, "Automatic classification of ships from infrared (FLIR) images", Proc. SPIE Defense and Security, vol. 3720, 1999. 